\title{
Bacterial vaginosis as a risk factor for high-grade cervical lesions and cancer in HIV-seropositive women
}

Sheri A. Denslow ${ }^{1}$, Daniel J. Westreich ${ }^{2}$, Cynthia Firnhaber ${ }^{3,4}$, Pam Michelow ${ }^{5}$, Sophie Williams ${ }^{4}$, and Jennifer S. Smith ${ }^{1,6}$

${ }^{1}$ Department of Epidemiology, Gillings School of Global Public Health, University of North Carolina, Chapel Hill, North Carolina

${ }^{2}$ Department of Obstetrics and Gynecology, Duke University School of Medicine and Duke Global Health Institute, Duke University, Durham, North Carolina

${ }^{3}$ Clinical HIV Research Unit Department of Medicine, Faculty of Health Sciences, University of Witwatersrand, South Africa

${ }^{4}$ Right to Care, Johannesburg, South Africa

${ }^{5}$ Cytology Unit, Department of Anatomical Pathology, Faculty of Health Sciences, University of the Witwatersrand and National Health Laboratory Service, Johannesburg, South Africa

${ }^{6}$ Lineberger Comprehensive Cancer Center, Chapel Hill, North Carolina

\section{Correspondence:}

Dr. Cynthia Firnhaber

Clinical HIV Research Unit Department of Medicine University of Witwatersrand Johannesburg, South Africa

Email: cindy.firnhaber@righttocare.org

Tel: 011 276-8860 | Fax : 011 276-8885

\section{Word count: 2,474}

\section{Short title: BV and high grade cervical lesions}

Key words: bacterial vaginosis, cervical lesions, HIV

Synopsis: Among HIV-seropositive women, bacterial vaginosis was not associated with an increased risk of HSIL or cervical lesion progression. 


\begin{abstract}
Objective: Both bacterial vaginosis (BV) and human papillomavirus (HPV) infections are common in HIV positive women. HPV/HIV coinfection is known to increase the risk of high-grade squamous intra-epithelial lesions (HSIL). The effect of BV on risk of HSIL, however, is unknown among HIVseropositive individuals.
\end{abstract}

Method: A hospital-based prospective cohort study of HIV-seropositive women was conducted in Johannesburg, South Africa from 2005 to 2009. Multivariate log-binomial and Poisson regressions were used to estimate prevalence and rate ratios, respectively.

Results: Among 1,954 HIV-seropositive women, the baseline prevalence of HSIL was 17\%. BV prevalence was high (54\%) and showed no association with prevalent HSIL (adjusted prevalence ratio: $1.12,95 \%$ CI: $0.92,1.35)$ nor with cervical lesion progression at follow-up visit $(\mathrm{N}=503)$ (adjusted rate ratio: $1.00,95 \% \mathrm{CI}: 0.65,1.53)$.

Conclusion: Among HIV-seropositive women, BV was not associated with an increased risk of HSIL or cervical lesion progression. 


\section{INTRODUCTION}

In 2008, an estimated 16 million women were living with HIV infection worldwide, the majority of which occurred in less-developed countries[1]. In South Africa, the 2008 estimated prevalence of HIV infection in the general population was $11 \%$. An estimated $60 \%$ of those cases were in women, with HIV prevalence as high as 33\% among women aged 20-34 years[2]. Regardless of HIV status, cervical cancer is the leading cause of cancer in African women, and the second most common in South African women[3]. Moreover, HIV-related immunosuppression is associated with a higher prevalence and incidence of precancerous cervical lesions as well as invasive cervical cancer [4]. The high prevalence of HIV in South African women combined with the high prevalence of precancerous high grade cervical lesions underscores the need to better understand the etiology of cervical cancer in this high-risk population.

Human papillomavirus (HPV) infection is well-documented as the central causal agent of cervical cancer. Most women infected with high risk HPV types, however, do not develop high grade cervical lesions and cancer[5]. Other factors acting in conjunction with HPV infection are likely involved in promoting the oncogenic capabilities of HPV, leading to the development of cancer. Coinfection of HPV with bacterial vaginosis (BV) has been suggested to advance this process and, for more than 25 years, has been considered a possible risk factor for cervical cancer development[6-16]. Only a few studies, however, have investigated the relationship between BV and cervical neoplasia in an HIV-seropositive study population, with inconsistent results $[10,16]$.

We therefore present here results of the association between BV and high grade squamous intra-epithelial lesions in a large survey of HIV-seropositive women from South Africa. 


\section{METHODS}

Study participants were enrolled in the South Africa Cervical Cancer Cohort (SACCC), an ongoing observational study. HIV-seropositive women, 18-65 years of age, were recruited from the Themba Lethu Clinic, an HIV outpatient clinic in a teaching hospital affiliated with the University of Witwatersrand in Johannesburg. All women consecutively enrolled between January 2005 and September of 2009 were included. Women were ineligible to participate in this study if they had undergone a hysterectomy or prior cone biopsy treatment for cervical lesions or were severely ill (per investigator judgment). Women with symptomatic STIs (e.g., excessive vaginal discharge) became study-eligible following treatment for the STIs; women who were pregnant became eligible 6 weeks after delivery.

Eligible women first attended an educational session on cervical cancer screening presented in English or Zulu, and those interested in participating gave written informed consent. All study protocols were reviewed and cleared by the University of Witwatersrand Human Research Ethics Committee and, for secondary data analysis, from the University of North Carolina. All study participants were treated according to the HIV South African Guidelines on Comprehensive HIV and AIDS Care, Management and Treatment, which initiates highly active antiretroviral therapy (HAART) at WHO stage 4 or CD4 count $<200$ cells $/ \mathrm{mm}^{3}$. Antibiotic treatment was offered to all women presenting with BV.

Medical history and potential risk factor information was obtained through interview at study entry and at follow-up visit. A medical exam, including a blood test, was performed to assess CD4 counts and the presence of symptomatic STIs. A pelvic exam was performed to collect samples for Pap analysis. For Pap analysis, cervical cells were collected using an endocervical brush. Conventional cervical smears were performed because liquid-based cytology is currently not available in the public health sector of South Africa. Cytology slides were read and analyzed 
according to the Bethesda 2001 reporting guidelines, categorized as negative for intraepithelial lesions or malignancy; atypical cells of undetermined significance (ASC-US); atypical squamous cells, cannot exclude high grade (ASC-H); atypical glandular cells (AGC); low-grade squamous intraepithelial lesions (LSIL); high-grade SIL (HSIL); or squamous cell carcinoma (SCC). For quality control, $10 \%$ of the cytology slides were sent to the University of North Carolina for a blinded second reading on two different occasions and a concordance of $81-85 \%$ was observed. The conventional cytology slides were also assessed for the presence or absence of clue cells, indicative of BV (Figure 1), as well as for trichomoniasis and candidiasis.

The outcome for cross-sectional analysis was the presence of HSIL, ASC-H, or SCC; collectively, this outcome was designated HSIL+. The non-outcome category included all other diagnoses, and was designated $<$ HSIL. The outcome for the analysis of rate of progression of cervical disease was any increase in grade of lesion, ordered from least to most serious in the following three categories: (1) negative; (2) LSIL, ASC-US, or AGC; and (3) HSIL, ASC-H, or SCC (collectively, HSIL+). Our main analysis categorized ASC-US and AGC results as LSIL, but we also explored categorizing these results as negative and excluding these results entirely.

There were two main analyses. First, we assessed the cross-sectional association of BV and HSIL+ status using prevalence ratios (PRs) and corresponding 95\% confidence intervals (CIs). Prevalence ratios were estimated with multivariable log-binomial regression.

Second we assessed the effect of baseline BV on rate of any cervical lesion progression (from negative to LSIL, negative to HSIL+ or from LSIL to HSIL+) by first follow-up visit. In addition, we assessed the effect of BV at baseline and at first follow-up visit, indicative of persistent BV, on cervical lesion progression. Progression analysis was restricted to women who were HSIL-negative at baseline, and to women whose first follow-up visit was at least six months after baseline; this latter condition was employed to exclude apparent progressions which were more likely to be measurement 
error, as insufficient time had passed for a true progression to occur. We estimated adjusted incidence rate ratios (IRRs) and 95\% CIs using Poisson regression with the log of follow-up time (time between visits) as the offset.

In both analyses, we identified potential confounding variables using directed acyclic graphs informed by literature. Covariates included CD4 count (categorized as $<200 ; 200-500$; and $>500$ cells $/ \mathrm{mm}^{3}$ ), trichomoniasis or candidiasis infection (yes/no), education level $(<$ grade 8; grades 8-12 years; > grade 12), unemployed (yes/no), current smoking (yes/no), current use of snuff (none; 1-3 /day; $>3$ /day), age of sexual debut $(<15 ; 15-18 ; 19-21 ;>21)$, lifetime number of sexual partners $(<5$; $5-10 ;>10)$, condom use at last act (yes/no), and age (included as a continuous linear and quadratic term).

In both regression modeling processes, covariates were maintained in the models through backwards elimination based on a likelihood ratio test $\mathrm{p}$-value of $<0.1$, although age and CD4 count were maintained in all models. Less than $4 \%$ of the study population had missing values for one or more covariates in the full multivariate model, and thus were excluded from final analyses. All analyses were performed using SAS 9.2 (SAS Corporation, Cary, NC).

\section{RESULTS}

A total of 1,954 women with baseline cytology contributed to analysis. Of these women, 1,063 (54\%) had BV at baseline (Table 1). Median age was 34 (range 18-65), and did not differ by baseline BV status. Most of the study population was of African ethnicity (98\%) with the remainder identifying as mixed African ethnicity (2\%). Approximately one-third of the women had matriculated from high school, with the majority receiving 8-12 years of education, and half (49\%) were unemployed. The median CD4 count for the population was 247 cells $/ \mathrm{mm}^{3}$ (interquartile range 
138-390 cells $/ \mathrm{mm}^{3}$ ), and was slightly lower for women with BV at baseline compared to women without BV at baseline. While $54 \%$ of the population had BV, trichomoniasis and candidiasis infections were relatively less common at $4 \%$ and $5 \%$, respectively. Few women reported current smoking (4\%) or snuff use (10\%). Most women reported no current hormonal contraceptive use (86\%), were between $15-18$ years of age at their sexual debut (61\%), had 4 or less lifetime partners (61\%), and reported current condom use (73\%).

The overall baseline prevalence of cervical lesions was $15 \%$ for LSIL and $17 \%$ for HSIL (Figure 2). ASC-US and ACG made up approximately $21 \%$ of the diagnoses, with the majority of these being ASC-US cases. Three cases of cervical cancer were observed; these women were treated immediately. Just under half of the women (47\%) had negative cytology at baseline. A $12 \%$ higher prevalence of HSIL + was observed among HIV-seropositive women with BV (adjusted PR: 1.12, 95\% CI: 0.92, 1.35, versus BV-negative), after adjustment for age, CD4 count, trichomoniasis or candidiasis infection, current hormone contraceptive use, and current smoking (Table 2).

A total of 503 women with $<$ HSIL diagnosed at baseline had a follow-up visit six or more months from their initial study visit. The median time for the follow-up visit was 1.2 years (IQR 1.1 to 1.4 years). Median follow-up time for women with and without BV at baseline was the same (1.2 years for both).

When including women with an ASC-US or AGC diagnosis as LSIL, 87 women showed progression. Women with BV at baseline were equally as likely as women without BV at baseline to show cervical lesion progression at their follow-up visit, after adjustment for CD4 count, age, and age at sexual debut (adjusted IDR: 1.00, 95\% CI:0.65, 1.53; shown in Table 3). A similar association was observed between persistent BV (women with BV at both baseline and first follow-up) and lesion progression. Women with persistent BV were 1.2 times as like as women without persistent BV to show cervical lesion progression at their follow-up visit, after adjustment for CD4 count, age, and age 
at sexual debut (adjusted IDR 1.18, 95\% CI:0.73, 1.92; data not shown). The effect estimates for BV at baseline as the exposure are slightly greater when women with ASC-US and AGC are grouped with negative cytology results (adjusted IDR: $1.27,95 \%$ CI: $0.76,2.11$ ) and also when women with ASC-US and AGC are excluded from the analysis entirely (adjusted IDR: 1.78, 95\% CI: 0.84, 3.79). However, these later results were statistically insignificant and less precise.

\section{DISCUSSION}

Among 1,954 HIV-seropositive women, prevalent BV was not associated with prevalent high grade cervical lesions or with the progression of cervical lesions at their next visit. Further, no association was seen between persistent BV and cervical lesion progression. These study results are distinctive in their focus on BV and high grade cervical lesions and cervical cancer within an HIVseropositive population in the resource-limited nation of South Africa.

A high prevalence of high-grade cervical lesions (17\%) was observed among these HIVseropositive women at baseline visit. This prevalence of HSIL is higher than that observed for HIVseropositive populations in France and French Guyana (7.5\%), a 12-nation, European cohort (2.8\%), Finland (5\%), and Zimbabwe (3.4\%)[10, 17-19], but lower than that found for an HIV-seropositive population in Zambia (32.6\%)[20]. Differences in HSIL prevalence across studies may be attributable in part to differences in HIV disease stage. Both our study population and the study group in Zambia were conducted among HIV-seropositive women with relatively lower median CD4 count (247 and 165 cells $/ \mathrm{mm}^{3}$, respectively) than that of the 12-nation European cohort $\left(378\right.$ cells $/ \mathrm{mm}^{3}$ ).

The lack of an association observed here for BV with high grade cervical lesions differs from the positive association found in a previous study in an HIV-seropositive population in Finland[10], and in populations from Brazil, Holland, and South Africa, in which HIV-status was unrecorded[7-9, 
14]. Our results are consistent, however, with those from two European studies with HIV status unknown[13, 15], and three studies in the US, two with unknown HIV-status[6, 12] and one with HIV-seropositive participants[16]. Four of these studies also assessed the association between BV and HPV infection, with only one[16] finding a positive association with HPV infection[6, 12, 15]. Although different methods were used to diagnose BV across studies (clinical diagnosis $(\mathrm{pH}>4.5$, positive amine test, positive gram stain, and characteristic vaginal discharge) $[9,12,13,15]$, gram stain[6, 16], or from Pap slides[7, 8, 10, 14]), in our view, the disparate association results do not appear to be explained by differences in methods to ascertain BV infection.

A high proportion of women surveyed had prevalent BV (54\%). Prevalence of BV has been shown to range from 5-35\% in the US and Europe, and from $18-70 \%$ in African nations[21]. Although there is debate as to if BV is a sexually transmitted infection (STI), BV is most common in women of reproductive age and has been associated with a higher number of reported sexual partners, the presence of other STIs, and less frequent condom use[22]. Estimates of association between BV and high grade lesions observed here was similar in the crude and multivariate analyses, suggesting that in this population, the association between BV and high grade cervical lesions is not notably confounded by shared demographic or sexual behavioral risk factors. Confounding by unmeasured factors, however, cannot be ruled out definitively.

It has been postulated that BV influences cervical lesion and cancer development both through an increase in vaginal nitrosamines and through a change in the vaginal cytokine profile[23]. Carcinogenic nitrosamines increase the probability of DNA damage and an altered cytokine profile may reduce the immune system's ability to eliminate HPV infection. Both changes may create an environment more conducive to cancer development. HIV-seropositivity also alters vaginal immune capabilities and cytokine profiles, further complicating the etiological mechanisms suggested to link BV to cervical precancer[24]. The lack of an association between BV and high grade cervical lesions 
observed here suggests a need to further consider the biological response to BV and its proposed relationship to cervical cancer in HIV-seropositive women.

The strengths of this study include its large size and its focus on an HIV-seropositive population in the resource-limited nation of South Africa. Few studies have addressed this vulnerable population and assessed the development and progression of precancerous lesions among HIVseropositive women. A key limitation of this study is the use of Pap slides for diagnosis of BV. The sensitivity for BV diagnosis from Pap slides ranges from 55-93\%, with specificity ranging from 94$98 \%$ as compared to gram stain assessment, the current gold standard in assessment[25]. Further limitations of the study include the use of a cytobrush for sample collection and the lack of histological confirmation for cytology results. Additionally, HPV status was not ascertained for participating women. Last, study participants were recruited from an HIV treatment center, thus limiting generalizability of the results to HIV-seropositive women able to seek treatment. However, study participants had a wide range in socioeconomic status markers of education and employment with close to $10 \%$ of the women receiving 8 or less years of school and $50 \%$ being unemployed. Plus, access to and obtainment of antiretroviral therapy is expanding rapidly in South Africa.

There is debate as to the benefits of treating asymptomatic BV in HIV-positive women in resource-limited settings. Based on the lack of association between BV and high grade cervical dysplasia and the lack of progression to high grade cervical dysplasia, treatment of asymptomatic BV may not be helpful in reducing the risk of HSIL development in this population. However further research is needed to evaluate BV and vaginal inflammation in the role of HPV and HIV transmission and disease course.

\section{Conflict of Interest}

The authors have no conflicts to disclose. 


\section{REFERENCES}

[1] UNAIDS/WHO. AIDS Epidemic Update: November 2009.

[2] Shisana O, Rehle T, Simbayi LC, Zuma K, Jooste S, Pillay-van-Wyk V, et al. South African national HIV prevalence, incidence, behaviour and communication survey 2008: A turning tide among teenagers? Cape Town: HSRC Press, 2009.

[3] GLOBOCAN. Cancer Incidence, Mortality and Prevalence Worldwide. Lyon, 2004.

[4] La Ruche G, Ramon R, Mensah-Ado I, Bergeron C, Diomande M, Sylla-Koko F, et al. Squamous intraepithelial lesions of the cervix, invasive cervical carcinoma, and immunosuppression induced by human immunodeficiency virus in Africa. Dyscer-Cl Group. Cancer $1998 ; 82(12): 2401-8$.

[5] Scheurer ME, Tortolero-Luna G, Adler-Storthz K. Human papillomavirus infection: biology, epidemiology, and prevention. Int J Gynecol Cancer 2005;15(5):727-46.

[6] Castle PE, Hillier SL, Rabe LK, Hildesheim A, Herrero R, Bratti MC, et al. An association of cervical inflammation with high-grade cervical neoplasia in women infected with oncogenic human papillomavirus (HPV). Cancer Epidemiol Biomarkers Prev 2001;10(10):1021-7.

[7] Discacciati MG, Simoes JA, Lopes ES, Silva SM, Montemor EB, Rabelo-Santos SH, et al. Is bacterial vaginosis associated with squamous intraepithelial lesion of the uterine cervix? Diagn Cytopathol 2006;34(5):323-5.

[8] Engberts MK, Verbruggen BS, Boon ME, van Haaften M, Heintz AP. Candida and dysbacteriosis: a cytologic, population-based study of 100,605 asymptomatic women concerning cervical carcinogenesis. Cancer 2007;111(5):269-74. 
[9] Kharsany AB, Hoosen AA, Moodley J, Bagaratee J, Gouws E. The association between sexually transmitted pathogens and cervical intra-epithelial neoplasia in a developing community. Genitourin Med 1993;69(5):357-60.

[10] Lehtovirta P, Paavonen J, Heikinheimo O. Risk factors, diagnosis and prognosis of cervical intraepithelial neoplasia among HIV-infected women. Int J STD AIDS 2008;19(1):37-41.

[11] McNicol P, Paraskevas M, Guijon F. Variability of polymerase chain reaction-based detection of human papillomavirus DNA is associated with the composition of vaginal microbial flora. J Med Virol 1994;43(2):194-200.

[12] Peters N, Van Leeuwen AM, Pieters WJ, Hollema H, Quint WG, Burger MP. Bacterial vaginosis is not important in the etiology of cervical neoplasia: a survey on women with dyskaryotic smears. Sex Transm Dis 1995;22(5):296-302.

[13] Uthayakumar S, Boyle DC, Barton SE, Nayagam AT, Smith JR. Bacterial vaginosis and cervical intraepithelial neoplasia--cause or coincidence? J Obstet Gynaecol 1998;18(6):572-4.

[14] Verbruggen BS, Boon ME, Boon LM. Dysbacteriosis and squamous (pre)neoplasia of immigrants and Dutch women as established in population-based cervical screening. Diagn Cytopathol 2006;34(5):377-81.

[15] Vetrano G, Pacchiarotti A, Lombardi G, Cimellaro V, Verrico M, Carboni S, et al. Correlation between squamous intraepithelial lesions (SILs) and bacterial vaginosis. Eur J Gynaecol Oncol 2007;28(4):310-2.

[16] Watts DH, Fazzari M, Minkoff H, Hillier SL, Sha B, Glesby M, et al. Effects of bacterial vaginosis and other genital infections on the natural history of human papillomavirus infection in HIV-1infected and high-risk HIV-1-uninfected women. J Infect Dis 2005;191(7):1129-39. 
[17] Chirenje ZM, Loeb L, Mwale M, Nyamapfeni P, Kamba M, Padian N. Association of cervical SIL and HIV-1 infection among Zimbabwean women in an HIV/STI prevention study. Int J STD AIDS 2002;13(11):765-8.

[18] Delmas MC, Larsen C, van Benthem B, Hamers FF, Bergeron C, Poveda JD, et al. Cervical squamous intraepithelial lesions in HIV-infected women: prevalence, incidence and regression. European Study Group on Natural History of HIV Infection in Women. AIDS 2000;14(12):177584.

[19] Six C, Heard I, Bergeron C, Orth G, Poveda JD, Zagury P, et al. Comparative prevalence, incidence and short-term prognosis of cervical squamous intraepithelial lesions amongst HIVpositive and HIV-negative women. AIDS 1998;12(9):1047-56.

[20] Parham GP, Sahasrabuddhe VV, Mwanahamuntu MH, Shepherd BE, Hicks ML, Stringer EM, et al. Prevalence and predictors of squamous intraepithelial lesions of the cervix in HIV-infected women in Lusaka, Zambia. Gynecol Oncol 2006;103(3):1017-22.

[21] Atashili J, Poole C, Ndumbe PM, Adimora AA, Smith JS. Bacterial vaginosis and HIV acquisition: a meta-analysis of published studies. AIDS 2008;22(12):1493-501.

[22] Fethers KA, Fairley CK, Hocking JS, Gurrin LC, Bradshaw CS. Sexual risk factors and bacterial vaginosis: a systematic review and meta-analysis. Clin Infect Dis 2008;47(11):1426-35.

[23] Pavic N. Is there a local production of nitrosamines by the vaginal microflora in anaerobic vaginosis/trichomoniasis? Med Hypotheses 1984;15(4):433-6.

[24] Nicol AF, Fernandes AT, Bonecini-Almeida Mda G. Immune response in cervical dysplasia induced by human papillomavirus: the influence of human immunodeficiency virus-1 coinfection -- review. Mem Inst Oswaldo Cruz 2005;100(1):1-12.

[25] Fitzhugh VA, Heller DS. Significance of a diagnosis of microorganisms on pap smear. J Low Genit Tract Dis 2008;12(1):40-51. 
Table 1. Sociodemographic, lifestyle, and medical characteristics of study participants at study entry in the South Africa Cervical Cancer Cohort

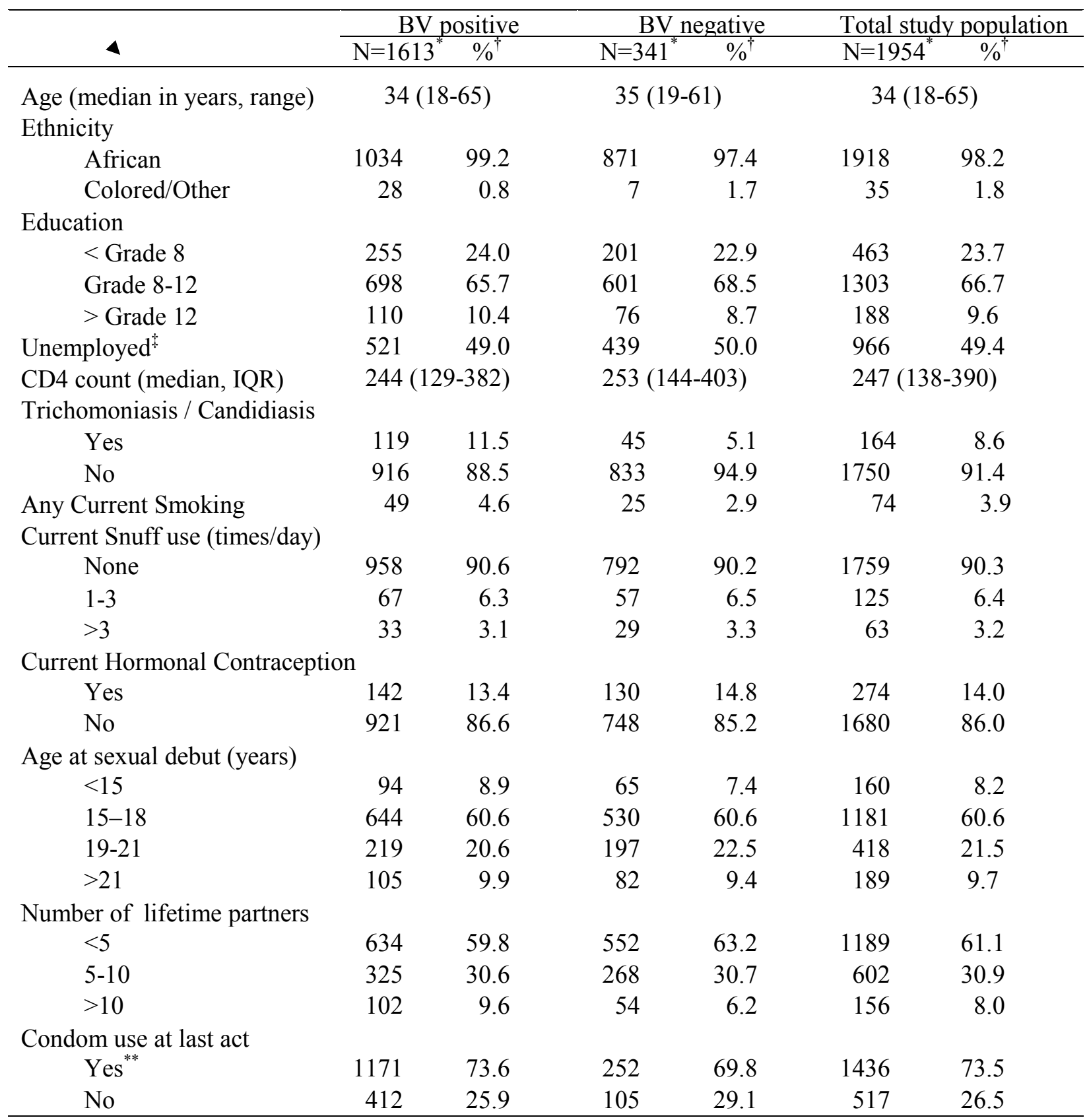

$\mathrm{IQR}=$ Interquartile range

* Numbers may not add up due to missing values

${ }^{\dagger}$ Percentages may not add to 100 due to rounding

*Employment includes students, pensioners and women on disability

** Includes women reporting abstaining from sex 
Table 2. Prevalence ratios of bacterial vaginosis and HSIL+ at study entry in the South Africa Cervical Cancer Cohort $(\mathrm{N}=1954)$

\begin{tabular}{lllll}
$\begin{array}{l}<\text { HSIL } \\
(\mathrm{N}=1613)\end{array}$ & $\begin{array}{l}\text { HSIL+ } \\
(\mathrm{N}=341)\end{array}$ & $\begin{array}{l}\text { Prevalence } \\
\text { of HSIL+ }\end{array}$ & Crude PR $(95 \% \mathrm{CI})$ & Adjusted PR ${ }^{*}(95 \% \mathrm{CI})$ \\
\hline & & & & \\
860 & 203 & $18.5 \%$ & $1.10(0.91,1.33)$ & $1.12(0.92,1.35)$ \\
725 & 153 & $15.7 \%$ & 1 & 1 \\
7 & 6 & & & \\
\hline
\end{tabular}

$\mathrm{PR}=$ prevalence ratio

* Model adjusted for CD4 count, age, current smoking, hormone contraceptive use, and prevalent trichomoniasis or candidiasis infections. 
Table 3. Incidence density ratio comparing progression of cervical lesions in women with BV at baseline to women without BV at baseline.

\begin{tabular}{|c|c|c|c|c|c|}
\hline & $\begin{array}{c}\text { Total } \\
\text { women }\end{array}$ & $\begin{array}{l}\text { Total follow-up } \\
\text { time (w-y) }\end{array}$ & Progressed ${ }^{*}$ & Crude IDR & Adjusted IDR ${ }^{\dagger}$ \\
\hline \multicolumn{6}{|c|}{ ASC-US/AGC as LSIL } \\
\hline $\mathrm{BV}+$ & 274 & 368.83 & 49 & $0.98(0.64,1.49)$ & $1.00(0.65,1.53)$ \\
\hline BV- & 229 & 279.76 & 38 & 1 & 1 \\
\hline \multicolumn{6}{|c|}{ ASC-US/AGC as negative } \\
\hline $\mathrm{BV}+$ & 274 & 368.83 & 39 & $1.23(0.74,2.05)$ & $1.27(0.76,2.11)$ \\
\hline BV- & 229 & 279.76 & 24 & 1 & 1 \\
\hline \multicolumn{6}{|c|}{ Excluding ASC-US/AGC } \\
\hline $\mathrm{BV}+$ & 190 & 262.40 & 27 & $1.85(0.87,3.94)$ & $1.78(0.84,3.79)$ \\
\hline BV- & 128 & 161.91 & 9 & 1 & 1 \\
\hline
\end{tabular}

$\mathrm{w}-\mathrm{y}=$ women-years, $\mathrm{IDR}=$ incidence density ratio

* Considered progression when repeat visit has cytology diagnosis that is higher than at baseline (negative to LSIL+, LSIL to HSIL+).

${ }^{\dagger}$ Final model adjusted for CD4 count, age, and age at sexual debut. 


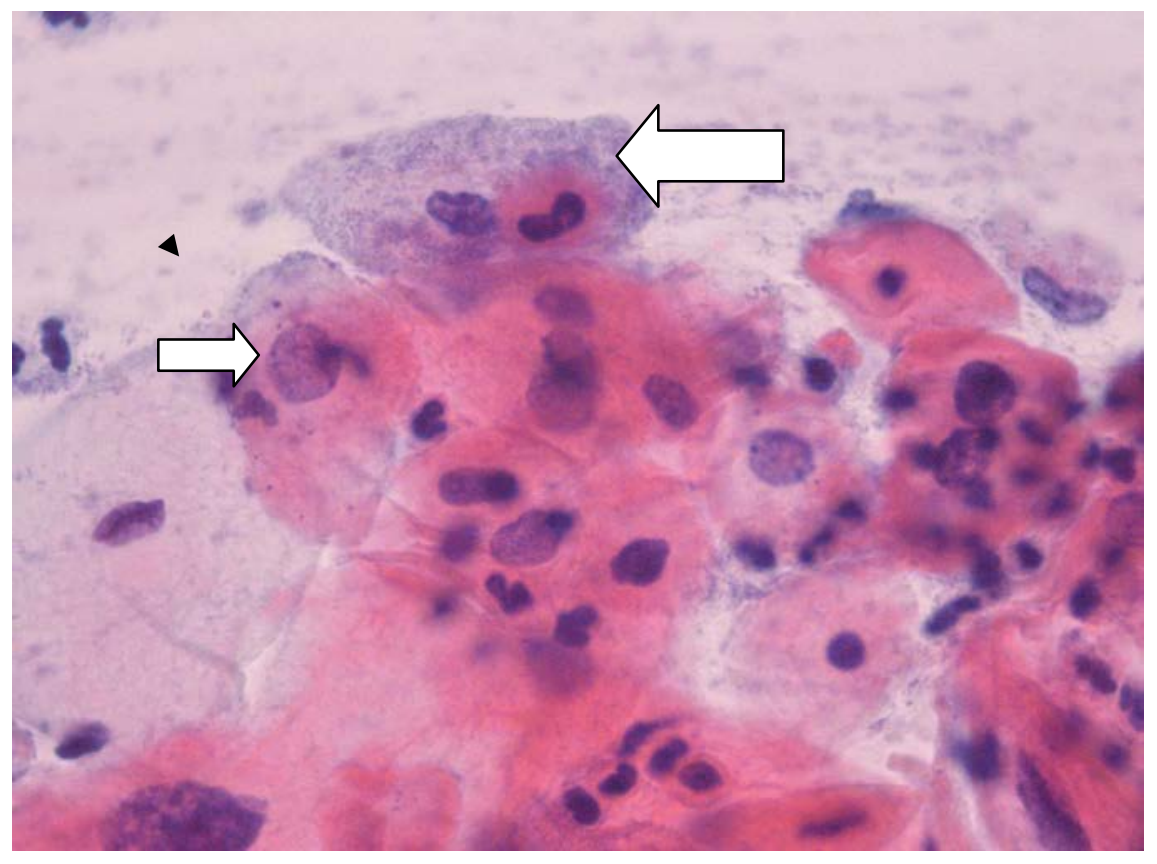

Figure 1: Cervical smear showing a 'clue cell' where the surface of the cell is covered with coccobacilli, consistent with bacterial vaginosis (large arrow). At smaller arrow, a cell consistent with a low grade squamous intraepithelial lesion, noted with nuclear enlargement and hyperchromasia (conventional smear, Papanicolaou stain x 400). 


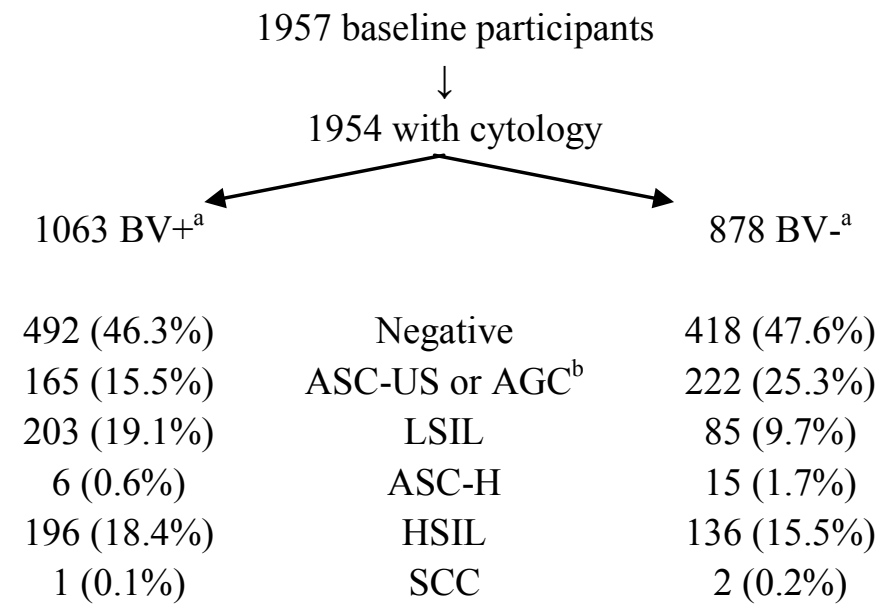

Figure 2. Pap smear diagnosis at study entrance in the South Africa Cervical Cancer Cohort, stratified by bacterial vaginosis prevalence

${ }^{\mathrm{a}} 13$ women were missing information on BV diagnosis

${ }^{\mathrm{b}}$ Of which 381 cases were ASC-US and 6 cases were AGC 